\title{
METODOLOGÍA DELPHI EN LA GESTIÓN DE LA INOCUIDAD ALIMENTARIA Y PREVENCIÓN DE ENFERMEDADES TRANSMITIDAS POR ALIMENTOS
}

\begin{abstract}
Carolina Palomino-Camargo 1,a, Yuniesky González-Muñoz ${ }^{1, b}$, Elevina Pérez-Sira ${ }^{1, c}$, Victor Hugo Aguilar ${ }^{2, d}$ RESUMEN

La presente revisión valora la metodología Delphi como técnica de consulta a expertos en el área Salud Pública, particularmente con un enfoque en la gestión de la inocuidad alimentaria y prevención de enfermedades transmitidas por los alimentos (ETA), brindándole a los investigadores, información metodológica para su uso. La compilación de la data se efectuó durante enero-agosto de 2017 en las bases de datos de Scopus, Medline y Scielo. Se evidenció que con los avances tecnológicos el método muestra un repunte, logrando obtener resultados en menor tiempo y costo de proceso, y un mayor alcance a expertos distantes, diversificándose su aplicabilidad en Inocuidad Alimentaria y prevención de ETA. Entre las principales aplicaciones destacan: diseño de sistemas de Análisis de Peligros y Puntos Críticos de Control (HACCP), herramienta ampliamente utilizada para lograr que los alimentos consumidos no ocasionen ningún daño al consumidor, descripción de variables, procesos y situaciones relacionados con las buenas prácticas de manufactura y condiciones sanitarias en la industria de producción y expendio de alimentos, útiles para la toma de decisiones.
\end{abstract}

Palabras clave: Testimonio de experto; Metodología; Salud pública; Enfermedades transmitidas por alimentos; Inocuidad alimentaria (fuente: DeCS BIREME).

\section{DELPHI METHODOLOGY IN FOOD SAFETY MANAGEMENT AND FOODBORNE DISEASE PREVENTION}

\begin{abstract}
This review assesses the Delphi methodology as an expert consulting technique in the area of Public Health, particularly with a focus on food safety management and prevention of foodborne diseases, providing researchers with methodological information for its use. The data was compiled during January-August 2017 in the databases of Scopus, Medline, and Scielo. It was evidenced that, with the technological advances, the method shows an upturn, obtaining results in less time and cost of process, and a greater reach to distant experts, diversifying its applicability in Food Safety and ATS prevention. Among the main applications are: design of Hazard Analysis and Critical Control Points (HACCP) systems, a tool widely used to ensure that the food consumed does not cause any harm to the consumer, description of variables, processes, and situations related to good manufacturing practices and sanitary conditions in the food production and retail industry, useful for decision making.
\end{abstract}

Keywords: Expert testimony; Methodology; Public health; Foodborne diseases; Food safety (source: MeSH NLM).

\section{INTRODUCCIÓN}

Las enfermedades transmitidas por los alimentos (ETA) son un problema de salud pública y una causa importante de morbilidad, que ocasiona una carga económica significativa para las naciones, perjuicios para los consumidores y un impacto al comercio internacional de productos alimenticios. Más de 250 enfermedades se transmiten a través de los alimentos y su incidencia ha aumentado considerablemente durante las últimas décadas por la globalización del mercado de alimentos y los cambios en los hábitos alimenticios ${ }^{(1-3)}$.

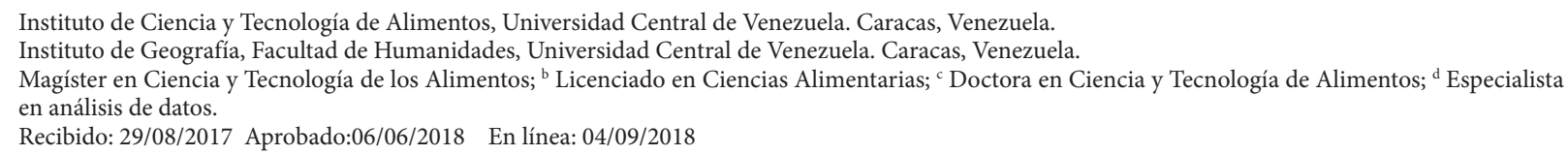

Citar como: Palomino-Camargo C, González-Muñoz Y, Pérez-Sira E, Aguilar VH. Metodología Delphi en la gestión de la inocuidad alimentaria y prevención de enfermedades transmitidas por alimentos. Rev Peru Med Exp Salud Pública. 2018;35(3): 483-90. doi: 10.17843/rpmesp.2018.353.3086. 
Adicionalmente, este problema se acrecienta con la aparición de nuevas formas de transmisión, en grupos poblacionales vulnerables, y el incremento de la resistencia bacteriana. Las infecciones humanas causadas por agentes patógenos (Ej. Salmonella spp., E. coli patogénica, Shigella, Listeria monocytogenes), las intoxicaciones provocadas por Staphyloccocus aureus, Clostridium botulinum, mohos productores de micotoxinas y las toxi-infecciones generadas por Bacillus cereus, Vibrio cholerae, Clostridium perfringens, entre otros, producen un gran número de muertes a nivel mundial $(2,3)$

Diversos estudios a nivel mundial han reportado casos de brotes por consumo de alimentos no inocuos, generado por manipulación incorrecta de los consumidores, fallas de los controles apropiados de calidad en los procesos de transformación, producción y servicios de expendio de alimentos, además, de errores en los programas de saneamiento (también conocidos como POES: Programa operacional estandarizado de saneamiento) y buenas prácticas de manufacturas (BPM) en la industria de los alimentos ${ }^{(1)}$

En tal sentido, se han diseñado programas de prevención de ETA y gestión de inocuidad de los alimentos, en los cuales se busca actuar y minimizar, desde un enfoque preventivo, los problemas asociados a la producción y manipulación de alimentos. Para ello, el sistema de análisis de peligros y puntos críticos de control, también conocidos como HACCP (del inglés Hazard Analysis and Critical Control Points), representa una herramienta de gran utilidad que es cada vez más exigida e implementada en los diversos rubros alimenticios producidos y comercializados ${ }^{(4,5)}$.

La base para la producción de alimentos inocuos, considerando este concepto como la garantía de que los alimentos no causen daño al consumidor cuando se preparen y/o consuman según el Codex Alimentario ${ }^{(6)}$, radica en las óptimas condiciones sanitarias y BPM. No obstante, la búsqueda constante de la inocuidad de los alimentos y prevención de ETA presenta innumerables desafíos en la actualidad y futuro. Por tanto, la constante gestión de la inocuidad de los alimentos y la prevención de las enfermedades transmitidas por estos, y cualquier iniciativa novedosa que busque minimizar riesgos asociados a las ETA, será bien valorada y se vuelve necesaria e importante para la agricultura, la industria alimentaria, los consumidores y la salud pública ${ }^{(2)}$.

Asimismo, la actualización y utilización constante del conocimiento teórico-práctico de los expertos en inocuidad e higiene de los alimentos, es un requisito para el desarrollo del saber y en ocasiones, una necesidad para el investigador y trabajador en esta área, el cual precisa apoyarse en la experticia de los colegas para dar respuesta a problemas surgidos o a prevenirlos ${ }^{(7,8)}$. Así, el método Delphi representa una metodología utilizada en la consulta de expertos ${ }^{(9)}$. En tal sentido, el presente artículo tiene como propósito brindar información que facilite el uso de la técnica Delphi, así como resaltar las principales aplicaciones en la prevención de las ETA e inocuidad alimentaria.

\section{METODOLOGÍA}

Se realizó una revisión narrativa con compilación de la información entre los meses de enero y agosto 2017. La búsqueda se llevó a cabo en las bases de datos Scopus, MEDLINE y SciELO con información comprendida entre losúltimos diezaños. Los términos de búsqueda aplicados para Scopus y MEDLINE fueron: «Delphi methodology», «foodborne disease», "food safety», «statistical procedure Delphi» y «advantages and disadvantages of Delphi». En el caso de SciELO se utilizaron los términos de búsqueda: «procedimiento Delphi», «consenso de expertos», «análisis estadístico de la consulta de expertos», "ETA», «inocuidad», «aplicaciones del Método Delphi en el sector alimento y tendencias». Se incluyeron artículos en inglés y español, así como referencias que hacían alusión al origen del método, evolución y actualidad.

\section{GESTIONANDO LA INOCUIDAD ALIMENTARIA Y LA PREVENCIÓN DE ETA MEDIANTE EL MÉTODO DELPHI: PASOS A SEGUIR}

Desde su aplicación, el método ha sido conceptualizado por investigadores, que propiamente lo han utilizado en sus líneas de trabajo, efectuando aportes significativos ${ }^{(10,11)}$. El método Delphi se caracteriza por ser una metodología estructurada para recolectar sistemáticamente juicios de expertos sobre un problema, procesar la información y construir un acuerdo grupal ${ }^{(7,12)}$. Para ello, se aprovecha la sinergia del debate en grupo y se eliminan las interacciones sociales indeseables que existen dentro del grupo; de esta forma se espera obtener un consenso lo más fiable posible del grupo experto ${ }^{(8,12)}$.

A medida en que fue creciendo la aceptación y el uso del método Delphi, precisamente por sus ventajas (Tabla 1), la herramienta también evolucionó en correspondencia a las nuevas necesidades que surgían, añadiendo así la creación de modalidades Delphi (Tabla 2). Estas modalidades, que parten del Delphi clásico, integran principios básicos que rigen el procedimiento, muy relacionado a la modalidad empleada: proceso iterativo, anonimato, retroalimentación y respuesta estadística del grupo ${ }^{(7)}$. Sin embargo, no existen requisitos estandarizados para el Delphi y se refleja gran variabilidad en relación a algunos parámetros (selección de expertos, características del cuestionario, número de rondas, procesamiento estadístico, definición del acuerdo general del grupo, entre otros). Esta variabilidad constituye una fortaleza que deriva de su adaptabilidad a las condiciones del estudio que se desarrolla ${ }^{(7)}$. 
Tabla 1. Descripción de las principales ventajas del método Delphi

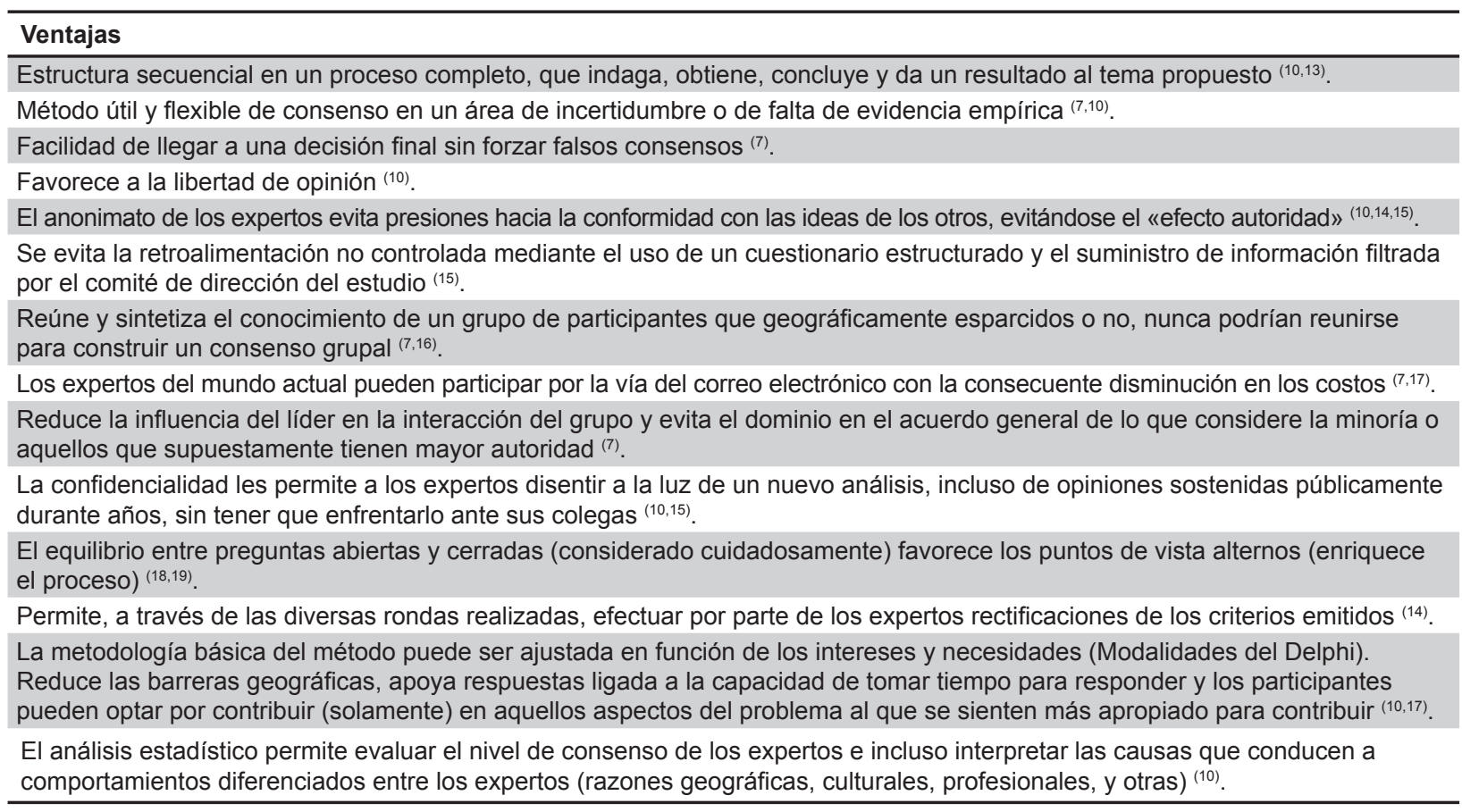

Una vez tomada la decisión de utilizarse el Delphi en la gestión de la inocuidad alimentaria y prevención de ETA, este conllevará a la ejecución de tareas progresivas, constituidas por una secuencia de acciones a lo largo de etapas, todas de gran trascendencia ya que aseguran la solidez metodológica y la calidad de los resultados. En tal sentido, se especifica tres etapas fundamentales: preparatoria (preliminar), exploratoria (de consulta), y final (de consenso) ${ }^{(7,10,12,19,26,27)}$. (Figura 1)

\section{APLICACIONES DEL MÉTODO DELPHI EN LA PREVENCIÓN DE ETA E INOCUIDAD ALIMENTARIA}

El método Delphi, representa una metodología que es ampliamente utilizada en los últimos cuarenta años en diversos campos: defensa, educación, agricultura, turismo y negocios ${ }^{(7,9,12)}$.

En la salud pública el método también ha tenido aceptación, evidenciando su aplicación en las diferentes áreas que engloban a este sector. No obstante, existen áreas con una mayor aplicación que en otras. En cuanto a su aplicación en la gestión de la inocuidad alimentaria y prevención de ETA, el método también muestra aprobación y evidencia gran aplicabilidad en estos dos temas relacionados con la salud pública y la producción/consumo de alimentos (Tabla 3). En la literatura se distingue el marcado uso de la metodología Delphi en el diseño de instrumentos que permiten evaluar la calidad e inocuidad en materia de producción y servicios de alimentos y en la validación de contenido (por expertos), específicamente en temas relacionados con inocuidad, seguridad alimentaria e higiene de los alimentos. Asimismo, se ha empleado en el análisis de peligros, dirigido al desarrollo del Sistema de Análisis de Peligros y Puntos Críticos de Control (HACCP), óptimo en la gestión de la inocuidad de los alimentos y prevención de ETA; así como en la búsqueda de estrategias y toma de acciones para garantizar el cumplimiento de las Buenas Prácticas de Producción de Alimentos, sobre todo en países en vías de desarrollo, mediante el avance de adecuados análisis de peligros, y para gestionar la calidad e inocuidad de los alimentos.

\section{DISEÑO DE INSTRUMENTOS PARA EVALUAR Y GESTIONAR LA CALIDAD E INOCUIDAD EN MATERIA DE PRODUCCIÓN Y SERVICIOS DE ALIMENTOS}

Delgado et al. ${ }^{(28)}$, realizaron un estudio mediante el método Delphi en mataderos ecuatorianos, partiendo de la premisa que, durante el sacrificio en dichas instalaciones, no se deben contaminar las canales con desechos y fluidos biológicos del animal y para garantizarlo se han establecido diversos documentos guías de BPM para el faenado, con el objetivo de hacer las actividades en el matadero más eficientes y obtener carnes de mayor calidad e inocuas. En tal sentido, con el fin de identificar las brechas existentes entre las condiciones en que se ejecuta el sacrificio y la obtención de las canales en mataderos ecuatorianos y las exigencias internacionales, se obtuvo una guía cuantitativa 


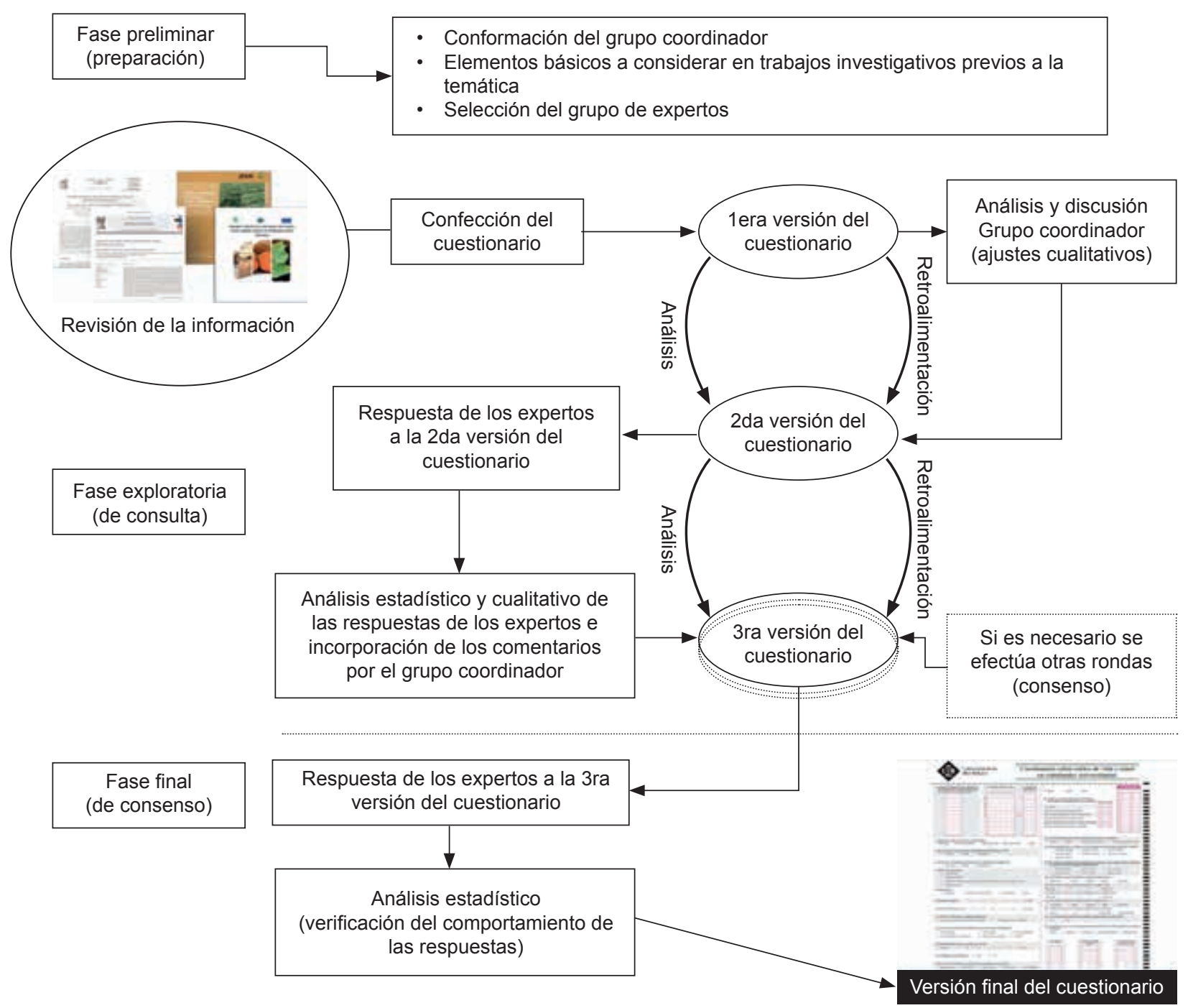

Figura 1. Representación esquemática de los pasos a seguir para aplicar la Metodología Delphi.

de evaluación, a través del uso de la técnica Delphi, de los requisitos de buenas prácticas de faenado con sus respectivas puntuaciones, destacando los elementos y subelementos que la conforman. La misma fue aplicada a varios mataderos, lo que permitió obtener una clasificación del nivel de implementación de las BPM de faenado en mataderos de diferentes zonas de Ecuador, en comparación a los criterios internacionales.

Por su lado, Soon et al. ${ }^{(29)}$, desarrollaron una herramienta de evaluación de riesgos de inocuidad alimentaria para granjas de salmón y productos frescos. En esta investigación el enfoque Delphi se utilizó para identificar y agregar las opiniones de expertos sobre los riesgos de inocuidad alimentaria y las enfermedades enfrentadas en las granjas, al mismo tiempo certificar los contenidos científicos de la herramienta. El cuestionario Delphi logró desarrollar acuerdo entre expertos sobre los riesgos y enfermedades asociados con las granjas productoras de salmón y productos frescos del Reino Unido, así como en relación a los temas utilizados en la herramienta de evaluación de riesgos de inocuidad alimentaria en granjas. La técnica Delphi demostró, a criterio de los autores, ser un enfoque valioso para agregar opiniones de múltiples expertos desde diversas regiones del mundo.

Mientras tanto, Jiun et al. ${ }^{(30)}$, realizaron una investigación para determinar los factores claves de éxito (FCE) de los proveedores de ingredientes de mariscos en la industria de la hospitalidad de Taiwán, relacionados con el HACCP. El panel de expertos (con experiencia de más de diez años en la implementación del HACCP) se utilizó para conectar e integrar los factores relacionados con los factores claves de éxito de los proveedores de ingredientes de mariscos. Los investigadores obtuvieron una tabla de evaluación para la selección de proveedores de ingredientes de mariscos en la industria de la hospitalidad, que puede ser utilizada como modelo en este país. 
Tabla 2. Modalidades de la metodología Delphi desde sus inicios prácticos

\begin{tabular}{|c|c|}
\hline Modalidad & Características \\
\hline $\begin{array}{l}\text { Delphi } \\
\text { político }\end{array}$ & $\begin{array}{l}\text { Fue propuesto en la década de } 1970 \text { y su } \\
\text { objetivo no es que un grupo tome una decisión, } \\
\text { sino que un grupo de expertos presenten } \\
\text { todas las opciones posibles ante un problema } \\
\text { y pruebas que sustenten los argumentos, en } \\
\text { lugar de tener un grupo que tome una decisión } \\
\text { consensuada }(10,20,21) \text {. }\end{array}$ \\
\hline $\begin{array}{l}\text { Delphi } \\
\text { Tradicional }\end{array}$ & $\begin{array}{l}\text { Consiste en el foro clásico para la priorización } \\
\text { de los hechos. Se compone de un cuestionario } \\
\text { enviado a un grupo de expertos, con un segundo } \\
\text { cuestionario basado en los resultados del primero. } \\
\text { Posteriormente, los cuestionarios se refinan y } \\
\text { definen los hechos o propuestas para medir la } \\
\text { exactitud o el soporte de los participantes }{ }^{(10,20,21)} \text {. }\end{array}$ \\
\hline \multirow{3}{*}{$\begin{array}{l}\text { Delphi } \\
\text { on-line }\end{array}$} & $\begin{array}{l}\text { Con el inicio del desarrollo de la informática, } \\
\text { se efectúan aportes a esta herramienta de } \\
\text { análisis, incorporando el término Delphi online } \\
\text { (una nueva modalidad para aquel entonces) y } \\
\text { ya en el } 2001 \text { se singulariza el método y se } \\
\text { habla de Cuestionario Delphi online }{ }^{(23)} \text {. }\end{array}$ \\
\hline & $\begin{array}{l}\text { El Delphi online (cuestionarios) retoma el } \\
\text { principio tradicional, pero, aunque puede reducir } \\
\text { en gran medida el tiempo y costo a través de la } \\
\text { utilización de la plataforma electrónica en relación } \\
\text { a la metodología convencional, no se tiene un } \\
\text { resultado inmediato (en corto tiempo) e implica } \\
\text { efectuar consecutivas rondas para lograr una } \\
\text { convergencia rápida (involucrando un lapso de } \\
\text { tiempo mucho mayor en el estudio) a diferencia } \\
\text { del Delphi en tiempo real }{ }^{(24,25)} \text {. }\end{array}$ \\
\hline & $\begin{array}{l}\text { A pesar de ello, el método continuó ajustándose a } \\
\text { la modernidad de las conexiones e interacciones } \\
\text { de las redes de internet y vía teléfonos } \\
\text { inteligentes, contribuyendo así al novedoso } \\
\text { concepto Plataforma Delphi en tiempo real }{ }^{(25)} \text {. }\end{array}$ \\
\hline $\begin{array}{l}\text { Delphi en } \\
\text { Tiempo-Real }\end{array}$ & $\begin{array}{l}\text { El Delphi en tiempo real se caracteriza por ser } \\
\text { una variante más corta donde el proceso se lleva } \\
\text { a cabo durante el curso de una reunión a través } \\
\text { de mecanismos para resumir las respuestas } \\
\text { dadas inmediatamente }{ }^{(10,20)} \text {. } \\
\text { Gordon y Pease (2006) plantean que esta } \\
\text { modalidad es ideal para una participación } \\
\text { sincrónica y mejorar la velocidad del proceso de } \\
\text { consulta, a tiempo real. Aunque se puede utilizar } \\
\text { también cuando la participación es asincrónica, } \\
\text { el número de participantes es mayor, y si se } \\
\text { dispone de más tiempo }{ }^{(25)} \text {. } \\
\text { Esta variante a su vez, puede soportarse en } \\
\text { plataformas que permiten la transformación } \\
\text { inmediata de los cuestionarios frente a la } \\
\text { retroalimentación lograda con los expertos y ofrece } \\
\text { al instante el resultado, mediante gráficos que } \\
\text { evidencian el comportamiento de las variables } \\
\text { evaluada (24). }\end{array}$ \\
\hline
\end{tabular}

Igualmente, Gutiérrez et al. (31), presentaron la construcción y validación inicial del instrumento GHYCAL (gestión de la higiene y calidad), aplicable en el diagnóstico de los siete planes previos de higiene y trazabilidad (Plan de control de agua, limpieza y desinfección, formación y control de manipuladores, mantenimiento preventivo, control de plagas
Tabla 3. Uso identificado del Método Delphi en la prevención de las enfermedades transmitidas por alimentos e inocuidad alimentaria.

\begin{tabular}{l}
\hline Aplicación general \\
Diseño de instrumentos que permitan evaluar y gestionar la \\
calidad e inocuidad en materia de producción y servicios de \\
alimentos. \\
Investigaciones asociadas \\
- Diseño de guías para las buenas prácticas de faenado en \\
mataderos (ponderación de los elementos de la Guía) ${ }^{(28)}$. \\
- Desarrollar y validar una herramienta para la evaluación de riesgos \\
de seguridad alimentaria en granjas (basado en expertos) \\
- Propuesta de una herramienta de evaluación para la selección \\
de proveedores de ingredientes (de mariscos) en la industria de \\
la hospitalidad que cumplan con el HACCP (30). \\
- Diseño de un instrumento para evaluar los niveles de gestión \\
de la higiene en empresas del sector agroalimentario (Validación \\
del contenido) (31).
\end{tabular}

- Análisis inicial de riesgo, dirigido al desarrollo de un HACCP ${ }^{(5)}$.

Aplicación general

Descripción de variables, procesos, situaciones, etc., que permiten la toma de decisiones.

\section{Investigaciones asociadas}

- Priorizar la gestión para la reducción de la contaminación por patógenos transmitido por alimentos en invernaderos de tomate en los Estados Unidos, mediante la determinación de las actividades y prácticas que probablemente contribuyen más a la contaminación microbiana y su mitigación ${ }^{(32)}$.

- Detectar los problemas que presenta una empresa cárnica en lo concerniente a la producción e inocuidad de los productos cárnicos que se elaboran, como elemento adicional utilizado para la Elaboración de un sistema de calidad HACCP mediante un diagnóstico funcional en el área de producción (33).

- Identificar el mínimo de formación inicial que debe darse a cada nuevo empleado con el fin de mantener al público a salvo de ETA, precisamente por la gravedad del riesgo de las ETA presentes en la industria de delicateses ${ }^{(34)}$

- Identificar y clasificar los comportamientos de manejo y consumo de alimentos asociados con 13 patógenos de alta incidencia de ETA.

- Definir procedimiento de trabajo para identificar los problemas emergentes de seguridad alimentaria en una etapa temprana e implicaciones en la práctica de gestión de riesgo internacional ( ${ }^{(36)}$.

- Priorizar la investigación para coordinar la gestión de la inocuidad de los alimentos y la calidad del agua (37).

- Obtener información de los manejos, normas, programas y resultados de programas, prevalencias y regulaciones con respecto a la problemática de Salmonella spp. y su visible predominancia dentro de los cuadros gastroentéricos humanos en Chile (38).

- Implementación de un sistema HACCP a partir de la correcta definición de los puntos críticos de control ${ }^{(4)}$.

HACCP: Sistema de análisis de peligros y puntos críticos de control; ETA: enfermedades transmitidas por alimentos.

y sistemas de vigilancia, control de la trazabilidad, y gestión de residuos), necesarios para implementar un protocolo de HACCP en empresas del sector agroalimentario. En particular, la metodología Delphi fue utilizada para realizar la validación del contenido (por expertos), específicamente en temas relacionados con inocuidad, seguridad alimentaria 
e higiene de los alimentos para el instrumento GHYCAL propuesto. Se validó su confiabilidad y criterio, resultando apropiado para la evaluación de los prerrequisitos del HACCP en empresas agroalimentarias.

Sin embargo, Hegelund y Sørensen ${ }^{(5)}$, utilizaron un panel de expertos para efectuar el análisis inicial de riesgo, dirigido también al desarrollo de un sistema de HACCP, pero con el fin de asegurar la salud y el bienestar de los animales en la producción ecológica de huevos. Basado en puntuaciones de severidad y ocurrencia, se seleccionó los diez principales problemas (el hambre, la sed, pilotes, el impacto de los cultivos, la cabeza negra, pasteurelosis, fracturas de huesos, el canibalismo, los depredadores y los ácaros rojos). Los expertos calificaron los factores de riesgo para esos problemas y finalmente indicaron puntos de control. Se seleccionaron 41 factores de riesgo que eran valorados con la más alta puntuación, considerando combinadamente su importancia y ocurrencia. Los autores, afirman que un panel de expertos, basado en cuestionarios, es una herramienta útil durante las primeras etapas del desarrollo de un plan de HACCP, específicamente en la realización de un análisis de peligros y puntos críticos de control.

\section{DESCRIPCIÓN DE VARIABLES, PROCESOS Y SITUACIONES PARA LA TOMA DE ACCIONES}

Asimismo, se observa la aplicación del Delphi en la descripción de problemáticas vinculadas a la reducción de la contaminación por patógenos transmitidos por alimentos y en establecer procedimientos de trabajo para definir los problemas emergentes, lo cual permitirá a futuro que las naciones puedan estar mejor preparadas frente a situaciones puntuales de brotes asociados a alimentos.

Siendo así, llic et al. ${ }^{(32)}$, consideraron como una amenaza constante para el público y la industria, el riesgo de contaminación y brotes severos de enfermedades en humanos, debido a patógenos transmitidos por los alimentos relacionados con productos frescos. Argumentan que existen herramientas limitadas para priorizar las prácticas de gestión de inocuidad de los alimentos en invernaderos comerciales y dirigidos a reforzar la inocuidad de los alimentos de manera oportuna como causa adicional del estudio. Por tal motivo, emplearon un enfoque Delphi para priorizar los factores manejados en la reducción de la contaminación por patógenos transmitidos por los alimentos en invernaderos de tomates. Con la finalidad de dar respuesta a la problemática, los investigadores ofrecieron a consulta una encuesta validada en línea que abordaba ocho áreas temáticas (gestión del agua, trabajadores, medioambiente y diseño de invernaderos, saneamiento de equipos, gestión de desechos, intrusión de animales, trazabilidad y propagación), la cual fue discutida entre 20 expertos internacionales reconocidos. Mediante la técnica Delphi determinaron clasificaciones de consenso de las actividades y prácticas que con mayor probabilidad contribuyen a la contaminación microbiana y su mitigación.

Cobo et al. ${ }^{(33)}$, utilizó adicionalmente el método con la finalidad de determinar puntos de control en el diseño de un sistema HACCP para una empresa de procesamiento de alimentos. Los autores llevaron a consulta tres preguntas cualitativas para luego evaluar los resultados en tres ocasiones. Para una última ronda las respuestas se convirtieron en resultados cuantitativos donde cada resultado fue tabulado mediante el diagrama de Pareto. De esta manera quedaron identificadas, en consenso, las etapas de procesamiento de los alimentos en la empresa que deben ser sumamente controladas. Bertolini et al. (4), en su caso, también emplearon el método Delphi con la finalidad de identificar puntos críticos de control del sistema HACCP en el procesamiento de alimentos para pequeñas y medianas empresas, pero abordaron los cuestionarios con mayor alcance, precisamente orientados a cómo los gerentes de calidad e inocuidad alimentaria pueden implementar objetiva y automáticamente los principios y el análisis de peligros del sistema HACCP.

Otro elemento importante en la prevención de las ETA y gestión de la inocuidad alimentaria lo constituye la capacitación del personal que labora en la producción y servicios de alimentos. En tal sentido, Kim et al. ${ }^{(34)}$, proponen identificar el mínimo de formación inicial que debe darse a cada nuevo empleado con el fin de mantener al público a salvo de enfermedades transmitidas por los alimentos (ETA), precisamente por la gravedad del riesgo de las ETA presentes en la industria de delicateses. Los objetivos de inocuidad alimentaria se obtuvieron en base al Código Alimentario de laAdministración deAlimentos y Drogas de los Estados Unidos (FDA) y cuatro plataformas de capacitación en inocuidad alimentaria en línea. Los resultados de esta investigación podrían servir, a título de los autores, como una guía cuando los instructores de inocuidad alimentaria en las operaciones de delicateses desarrollan sus propios materiales de capacitación o identifican conceptos clave en las plataformas de formación existentes. No obstante, Hillers et al. (35) , enfocaron su estudio en la identificación y clasificación de los comportamientos de consumo y manipulación de alimentos asociados a los principales patógenos transmitidos por alimentos. El ranking de comportamientos resultante por el panel experto para reducir el riesgo de enfermedades causadas por patógenos alimentarios, puede permitirles a los consumidores tomar decisiones informadas sobre el consumo y manipulación de los alimentos, al igual que serviría de guía a los capacitadores de la inocuidad alimentaria en la priorización de los esfuerzos educativos.

Muchos de los estudios anteriormente citados favorecen a la toma de decisiones. Sin embargo, con un alcance global, Marvin et al. (36), utilizó una encuesta mediante el Delphi sobre el análisis de riesgos de inocuidad de los alimentos, revelando preocupaciones en varios aspectos de los procedimientos actuales para tratar problemas emergentes. 
Los encuestados en el Delphi también otorgaron un gran valor a la participación de las partes interesadas y la inclusión de una gama más amplia de datos en el análisis de riesgos. De hecho, considerando los resultados de este estudio y criterios adicionales de otros expertos, actualmente se están desarrollando sistemas holísticos que emplean indicadores externos a la cadena de producción de alimentos. Como resultado significativo se destaca la propuesta de un procedimiento de cuatro pasos para la identificación temprana de problemas emergentes ${ }^{(36)}$.

Crohn y Brianchi priorizaron la técnica para coordinar la gestión de la inocuidad de los alimentos y la calidad del agua en granjas que cultivan lechuga y vegetales de hojas verdes, mediante regadío, en la costa central de California y que ponen en conflicto la calidad del agua superficial (37). Los expertos participantes (en calidad del agua e inocuidad alimentaria) conceptualizaron los problemas de manera diferente, aunque no hubo desacuerdo profundo en las necesidades de investigación.

Figueroa y Urcelay realizaron un estudio con la finalidad de describir la situación de la salmonelosis humana y animal en Chile en el periodo 2000-2006 y comparar la aplicación de buenas prácticas con la incidencia de salmonelosis humana (38). Aplicaron un cuestionario Delphi de 33 preguntas a expertos, las cuales fueron divididas para su mejor comprensión en cinco temas: preguntas de carácter general, epidemiología, producción animal, ETA y salud humana. La visión de los expertos pone a la salmonelosis como una patología que se presenta con mayor frecuencia en meses de verano y que afecta por igual a hombres y mujeres especialmente en la zona central de Chile. Asumieron también que la salmonelosis es un problema grave, tanto nacional como internacionalmente, cuyo principal contagio se produce al interior del hogar. Los alimentos de mayor relevancia, según los expertos en el contagio de Salmonella spp. son los huevos y sus derivados. Plantearon que la bacteria no puede ser eliminada de las granjas avícolas debido a sus vías de transmisión y resistencia, por lo cual el esfuerzo mayor debe ser puesto en bajar su prevalencia y en educar al consumidor para un buen almacenamiento y preparación de los productos de alto riesgo. Finalmente, coincidieron en que es necesaria una organización que reúna personal experto de salud pública y animal, aplicando buenas prácticas de producción en toda la cadena, para poder combatir esta patología y así disminuir su prevalencia.

\section{CONCLUSIONES}

El método Delphi representa una herramienta de gran utilidad en el área de prevención de ETA e inocuidad alimentaria, se evidencia su aplicabilidad en escenarios futuros. La evolución del método y sus modalidades, cada vez más adaptadas a las necesidades que se imponen, con particular interés en el Delphi online y Delphi en tiempo real, ofrecen un abanico de oportunidades para la cadena de producción de alimentos y la salud pública.

El método evidencia ventajas que fortalecen a esta herramienta, las más destacadas son: técnica grupal de análisis de opinión; reúne y sintetiza el conocimiento de un grupo de participantes geográficamente esparcidos o no, con diferentes experiencias culturales y puntos de vista que favorecen de una manera interesante la generación de ideas y expectativas similares para el futuro; el análisis estadístico permite evaluar el nivel de consenso de los expertos e incluso interpretar las causas que conducen a comportamientos diferenciados entre los expertos; el anonimato de los expertos evita presiones hacia la conformidad con las ideas de los otros, impidiéndose el "efecto autoridad"; permite la transformación durante la investigación de las apreciaciones individuales de los expertos en un juicio colectivo superior. Estos elementos fortalecen y dinamizan el arsenal de aplicabilidad del método en la investigación, desarrollo e innovación (I+D+I) actual y futuro.

Los usos identificados, se pueden organizar en dos grandes grupos: diseño de instrumentos y sistemas que garanticen las buenas prácticas higiénicas y la inocuidad de los alimentos durante su producción y/o elaboración (Ej. Sistemas HACCP); así como en la descripción de variables, procesos, situaciones que permitan la toma de acciones.

Contribuciones de los autores: CPC, YGM, EPS, VHA participaron en la concepción, diseño del artículo, redacción y aprobación final.

Fuentes de financiamiento: Autofinanciado.

Conflictos de interés: Los autores declaran no tener conflictos de interés con el presente artículo.

\section{REFERENCIAS BIBLIOGRÁFICAS}

1. González Y, Palomino C, Calderín A. El perfil sanitario como una herramienta para la gestión de la calidad higiénica e inocuidad de los alimentos (caso restaurante). Revista Observador del Conocimiento. 2014;2(4):105-18.

2. PalominoC,González Y.Técnicasmoleculares parala detección eidentificación de patógenos en alimentos: ventajas y limitaciones. Rev Peru MedExpSalud Publica. 2014;31(3):535-46.

3. González Y, Palomino C. Acciones para la gestión de la calidad sanitaria e in- 
ocuidad de los alimentos en un restaurante servicio bufet. Rev Gerenc Polit Salud. 2012;11(22): 123-40.

4. Bertolini M, Rizzi A, Bevilacqua M. An alternative approach to HACCP system implementation.J FoodEng. 2007;79(4):1322-28. 5. Hegelund L, Sorense J. Developing a HACCP-like system for improving animal health and welfare in organic egg production - based on an expert panel analysis. Animal. 2007;1(7):1018-25.

6. Codex Alimentarius. Higiene de los Alimentos. Textos básicos. Código Internacional recomendado de Prácticas. Principios generales de higiene de los alimentos. FAO/OMS, Roma, Italia; 2003 [citado el 15 de agosto de 2017] Disponible en: http://www.fao.org/docrep/005/Y1579S/ y1579s00.htm

7. García M, Suárez M. El método Delphi para la consulta a expertos en la investigación científica. Rev Cub de Salud Pública. 2013;39(2):253-67.

8. Landeta J, Matey J, Ruíz V, Villarreal O. Alimentación de modelos cuantitativos con información subjetiva: aplicación del Delphi en la elaboración de un modelo de imputación del gasto turístico individual en Catalunya. Questiió. 2002;26(1-2):175-96.

9. Cruz M, Campano A. El procedimiento de la información en investigaciones educacionales. 1a ed. La Habana: Educación Cubana; 2008

10. Varela M, Díaz L, García R. Descripción y usos del método Delphi en investigaciones del área de la salud. Inv Ed Med. 2012;1(2):90-5.

11. González Y, Pérez E. Diseño, validación e implementación de un instrumento para determinar la precepción del consumo sobre raíces y tubérculos en estudiantes universitarios. [Tesis de maestría]. Venezuela: Instituto de Ciencia y Tecnología de Alimentos, Universidad Central de Venezuela, 2017.

12. Aponte F, Cardozo M, Melo R. Método Delphi: aplicaciones y posibilidades en la gestión prospectiva de la investigación y desarrollo. Revista Venezolana de Análisis de Coyuntura. 2012;18(1):41-52.

13. Diamond I, Grant R, Feldman B, Pencharz P, Ling S, Moore A, et al. Defining consensus: A systematic review recommends methodologic criteria for reporting of Delphi studies. J Clin Epidemiol. 2014;67(4):401-9.

14. Holloway K. Doing the E-Delphi: using online survey tools. Computer, informatics, Nursing. 2012;30(7):347-50.

15. Steurer J. The Delphi method; an efficient procedure to generate knowledge. Skeletal Radiol. 2011;40(8):959-61.
16. Boulkedid R, Abdoul H, Loustau M, Sibony $\mathrm{O}$, Alberti $\mathrm{C}$. Using and reporting the Delphi method for selecting healthcare quality indicators: a systematic review. PloS One. 2011;6(6):e20476.

17. Bennassar M. estilo de vida y salud en estudiantes universitarios; la Universidad como entorno promotor de salud. [Tesis doctoral]. Universitat de les IIIes Baleares. 2011.

18. Bravo M, Arrieta J. El método Delphi. Su importancia en una estrategia didáctica para la enseñanza de las demostraciones geométricas. Rev Iberoamericana de Educación. 2005;36(7):1-10.

19. Velásquez $S$, Castro J. Identificación de factores de éxito para el sector cuero, calzado y marroquinería en Colombia, usando metodología Delphi: análisis estructural y juego de actores. Informador técnico. 2013;77(2):136-46.

20. De Villiers M, De Villiers P, Athol K. The Delphi technique in health sciences education research. Med Teach. 2005;27(7):639-43.

21. Turoff M. The design of a policy Delphi. Technol Forescast Soc Chang. 1970;2(2):149-71.

22. Linstone H, Turoff M. Introduction. En Linstone HA, Turoff M, editores. The Delphi Method: Techniques and applications. Londres: Addison-Wesley Publishing Co. Inc.; 2002. p. 5-12.

23. Moldrup C, Morgall J. Risks of Future Drugs: A Danish Expert Delphi. Technol Forecast Soc Change. 2001;67(2-3):273-89.

24. Gnatzy T, Warth J, der Gracht $H$, Inga D. Validating an innovative real-time Delphi approach - A methodological comparison between real-time and conventional Delphi studies. Technol Forecast Soc Change. 2011;78(9):1681-94.

25. Gordon T, Pease A. RT Delphi: An efficient, round-less almost real time Delphi method. Technol Forecast Soc Change. 2006;73(4):321-33.

26. Domínguez M, Gómez F. Pagos agroambientales y productores agrarios: un análisis Delphi de las percepciones y demandas de los ganaderos beneficiarios de los programas de ayudas. Revista Española de Estudios Agro-sociales y Pesqueros. 2013;236:81-118

27. Pérez L, Llorente E, Gavidia V, Caurín C, Martínez M. ¿Qué enseñar en la educación obligatoria acerca de la alimentación y la actividad física? Un estudio con expertos. Enseñanza de las Ciencias. 2015;33(1):85-100.

28. Delgado H, Roque E, Alfredo C, Villoch A. Análisis del cumplimiento de las buenas
Prácticas de faenado en cinco mataderos municipales de Manabí, Ecuador. Rev Salud Anim. 2015;37(2):69-78.

29. Soon J, Davies W, Chadd S, Basines R. A Delphi-based approach to developing and validating a farm food safety risk assessment tool by experts. Expert Syst Appl. 2012; 39(9):8325-36.

30. Jiun $\mathrm{H}, \mathrm{Fu} W, \mathrm{Kaie} C h$. Research on key success factors of seafood ingredient suppliers in hospitality industry of Taiwan. Actual problems of Economics. 2011;123(9):383-93.

31. Gutiérrez N, Pastrana E, Ramírez E. Desarrollo de un instrumento para evaluar prerrequisitos en el sistema Haccp. Rev Bio Agro. 2010;8(1):106-19.

32. IlicS, LeJeune J,Lewis M, MillerS. Delphiexpert elicitation to prioritize food safety management practices in greenhouse production of tomatoes in the United States. Food Control. 2017;78:108-15. doi: https://doi org/10.1016/j.foodcont.2017.02.018

33. Cobo C, Alcívar P, Saltos J. Elaboración de un sistema de calidad HACCP mediante un diagnóstico funcional en el área de producción de la empresa SUMERCO S.A. [Tesis de pregrado]. Ecuador: Escuela Superior Politécnica Agropecuaria de Manabí, Manuel Félix López; 2016. 34. Kim K, O’Bryan C, Crandall P, Ricke $S$, Neal J. Identifying baseline food safety training practices for retail delis using the Delphi expert consensus method. Food Control. 2013;32(1):55-62.

35. Hillers V, Medeiros L, Kendall P, Chen G, Dimascola S. Consumer Food-Handling Behaviors Associated with Prevention of 13 Foodborne Illnesses. J Food Prot. 2003;66(10):1893-99.

36. Marvin H, Kleter G, Frewer L, Cope S, Wentholt M, Rowe G. A working procedure for identifying emerging food safety issues at an early stage: Implications for European and international risk management practices. Food Control. 2009;20(4):345-56.

37. Crohn D, Bianchi M. Research Priorities for Coordinating Management of Food Safety and Water Quality. J. Environ. Qual. 2008;37(4):1411-18.

38. Figueroa J, Urcelay S. Descripción y análisis de las acciones realizadas por los servicios públicos (salud animal y salud pública), frente a salmonelosis humana. [Tesis de pregado]. Chile: Facultad de Ciencias Veterinarias y Pecuarias, Escuela de Ciencias Veterinarias, Universidad de Chile, Santiago de Chile; 2007.

Correspondencia: Yuniesky González Muñoz. Dirección: Instituto de Ciencia y Tecnologia de Alimentos, Facultad de Ciencias, Universidad Central de Venezuela. Caracas, Venezuela Correo electrónico:yunieskygm@yahoo.es 